\title{
Natural Analogs of Synthetic Reference Materials: Assessing the Limits of Homogeneity Testing at the Picogram Scale
}

\author{
P.P. Michalak, ${ }^{*}$ A.D. Renno, ${ }^{*}$ S. Merchel, ${ }^{* *}$, F. Munnik, ${ }^{* *}$ and M. Wiedenbeck*** \\ * Institute of Mineralogy, TU Bergakademie Freiberg, D09596 Freiberg, Germany \\ ** Helmholtz-Zentrum Dresden-Rossendorf, D01328 Dresden, Germany \\ *** Helmholtz-Zentrum Potsdam Deutsches GeoForschungsZentrum, D14473 Potsdam, Germany
}

The demand for certified reference materials for chemical and structural microanalysis of geological samples is growing continuously. The fact that there are no certified materials currently available, for which the chemical homogeneity has been documented at the micrometer- and submicrometer level [1] reinforces the need of producing them. A collaboration involving several German scientific institutions has now received funding from the Free State of Saxony and the European Union Social Fund with the objective of providing synthetic, adequately homogeneous minerals with appropriate contents of economically important trace elements that could be certified as reference materials for methods employing sub-ng sampling masses.

Due to such geological processes as crystallization, metasomatism and weathering, specific elemental exchange vectors and substitution mechanisms may be triggered $[2,3]$ (as shown in Fig. 1), causing elements in minerals to be spatially, heterogeneously distributed at the micrometer scale [4] (e.g. zonation, see Fig. 2). An awareness of naturally occurring solid solutions as well as different structural characters in the lattice of minerals must, therefore, form a foundation for any attempt at producing synthetic mineral materials, which according to this project's objective, should be free of any compositional and structural inhomogeneities at such a sampling scale.

Prior to the synthesis of any minerals, a thorough crystal-chemical characterization of their natural analogs should be performed. A certain spatial variation in the concentration of major and trace elements in natural mineral samples may be revealed using microanalytical techniques of varied sensitivity. One way to perceive homogeneity is as the function of the size of the studied analytical volume, which is largely determined by the spatial resolution of the probe. For example, a material rated homogenous by optical microscopy and EPMA may demonstrate inhomogeneity when tested using nanoSIMS because of the better spatial resolution and detection limits provided by this method. On the other hand, an isotopically homogenous material may exhibit heterogeneity in the major and/or trace element distributions [5].

In order to test such variances and relationships in selected natural analogs of synthetic reference materials as a function of analytical method, we designed a series of chemical and structural homogeneity-check measurements of sanidine, tantalite, columbite and pyrite. We are using several different imaging and spectroscopic techniques, starting from optical polarizing and reflected microscopy, BSE and CL imaging, EBSD, IR, Raman, EPMA, $\mu$ PIXE coupled with $\mu$ PIGE, SIMS and synchrotron $\mu \mathrm{XRF}$. 
Establishing a rapid, economical and robust homogeneity testing procedure is essential for achieving the overall goals of this project. In order to meet these criteria, a reasonable sequence of measurements is devised starting from simple techniques (i.e., optical microscopy) and move toward the more complex (i.e. SIMS and $\mu \mathrm{XRF}$ ). This first phase will contribute towards optimizing our homogeneity testing procedure, assuring that our approach will be suitable for our ultimate certification plan.

\section{References}

[1] M. Wiedenbeck, Goldschmidt Conference Abstracts 2010, A1130.

[2] S. Spear, Metamorphic Phase Equilibria and Pressure-Temperature-Time Paths. MSA, Washington D.C., 1993.

[3] T. S. Ercit et al., Can. Mineral. 32 (1994) 421-438.

[4] L. Nasdala et al., Chem. Geol. 269 (2010) 290-300.

[5] A. K. Kennedy et al., Can. Mineral. 48 (2010) 1423-1443.

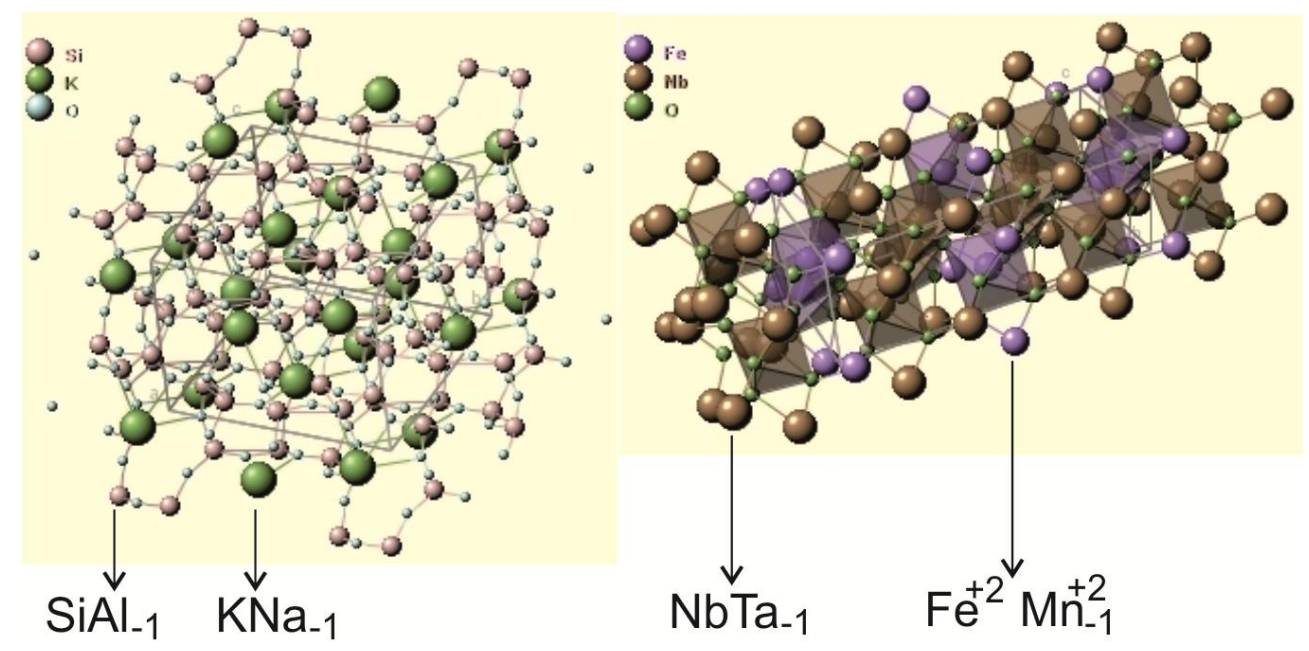

FIG. 1. Elemental exchange vectors in sanidine (left) and columbite (right). Schematics adapted from www.webmineral.com.
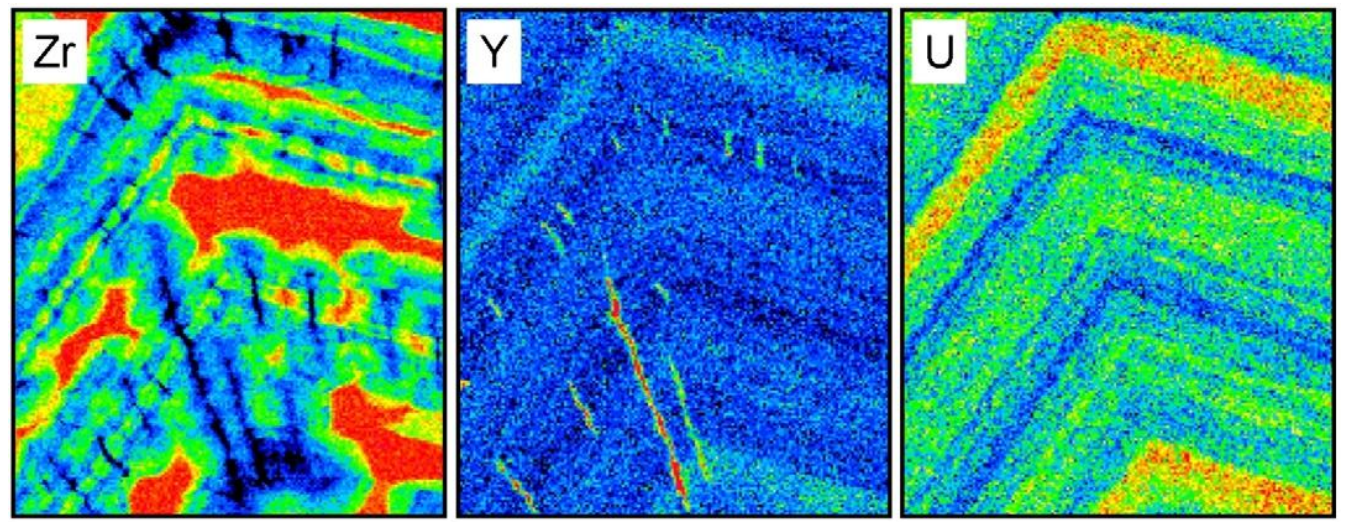

FIG. 2. EPMA element maps of altered zircon showing fine zonation as well as uranium distribution pattern independent of zirconium and yttrium [4]. 